COMMUNICATIONS IN

ANALYSIS AND GEOMETRY

Volume 11, Number 3, 553-564, 2003

\title{
The Lefschetz theorem for CR submanifolds and the nonexistence of real analytic Levi flat submanifolds
}

\author{
LEI Ni AND JON WOLFSON
}

\section{Introduction.}

In this paper we study the topology of a CR submanifold with degenerate Levi form embedded in a compact Hermitian symmetric space. In the case that the ambient manifold is $\mathbb{P}^{v}$ we prove a Lefschetz hyperplane theorem for such CR submanifolds. In addition we relate the homotopy groups of the ambient manifold with the homotopy groups of the CR submanifold within a range depending on the nullity of the Levi form. The optimal results are obtained for Levi flat submanifolds. In particular we show that a compact Levi flat submanifold in a compact Hermitian symmetric space of complex dimension $v$ is simply connected if the real dimension of the Levi flat manifold is greater than $v+1$. We then apply this result to give a proof of the nonexistence of real analytic Levi flat submanifolds of real dimension greater than $v+1$. The proof of the Lefschetz theorem is based on the Morse theory of paths between a CR submanifold and a complex submanifold. The proof of the other restrictions on the topology of a CR submanifold is based on the Morse theory of paths between two CR submanifolds. The crucial ingredient in both proofs is a second variation calculation in Schoen-Wolfson $[\mathrm{S}-\mathrm{W}]$, that goes back to Frankel $[\mathrm{F}]$.

To our knowledge results of this type relating the topology of a CR submanifold with its Levi form are the first of their kind. They are natural generalizations of analogous results for complex submanifolds. On the nonexistence of Levi flat submanifolds: Lins Neto proved the nonexistence of real analytic Levi flat hypersurfaces in $\mathbb{P}^{v}$, for $v \geq 3[\mathrm{LN}]$; Ohsawa [O] did the $v=2$ case, and Siu [S 1, S 2] proved the nonexistence of smooth Levi flat hypersurfaces in $\mathbb{P}^{v}$. Whether our nonexistence result remains true in the smooth category is an interesting open problem.

\footnotetext{
${ }^{1}$ The first author was partially supported by NSF grant DMS-0203023. The second author was partially supported by NSF grant DMS-0104007. Both authors wish to thank the National Center for Theoretical Sciences, National Tsing Hua University, Hsinchu, Taiwan for the hospitality provided during the writing of parts of this paper.
} 


\section{Definitions and the statements of the theorems.}

We will use the following notion defined in [K-W]: Let $V$ be an irreducible compact Kähler manifold of complex dimension $v$ with nonnegative holomorphic bisectional curvature. Define the symmetric bilinear form $H_{Y}(W, Z)=\langle R(Y, J Y) W, J Z\rangle$, where $Y, W, Z \in T V$. Then for any $Y \neq 0$, $H_{Y}$ is positive semi-definite. Denote the null-space by $\mathcal{N}_{Y}$. Note that $\mathcal{N}_{Y}$ is a complex vector space. Let $n(Y)$ be the complex dimension of $\mathcal{N}_{Y}$ and set $\ell(Y)=v-n(Y)$. Define the complex positivity $\ell$ of $V$ by

$$
\ell=\inf _{Y \neq 0} \ell(Y) \text {. }
$$

By the classification result of Mok [M], the above definition is independent of the choice of the base point. An equivalent definition is used in $[\mathrm{K}-\mathrm{W}]$ where $\ell$ is computed for all compact Hermitian symmetric spaces. In particular in $[\mathrm{K}-\mathrm{W}]$ it is shown:

$$
\begin{array}{ll}
\mathbb{P}^{v}: & \ell=v . \\
\mathbf{G r}(p, p+q ; \mathbb{C}): & \ell=p+q-1 . \\
\mathbf{G r}(2, p+2 ; \mathbb{R}): & \ell=p-1 . \\
\operatorname{Sp}(r) / U(r): & \ell=r . \\
\operatorname{SO}(2 r) / U(r): & \ell=(r-1)+(r-2) . \\
E_{6} /\left(\operatorname{Spin}(10) \times T^{1}\right): & \ell=11 \\
E_{7} /\left(E_{6} \times T^{1}\right): & \ell=17 .
\end{array}
$$

This computation goes back to $[\mathrm{B}]$ and $[\mathrm{C}-\mathrm{V}]$. It is also related to the concept called degree of nondegeneracy of the bisectional curvature in the Kähler geometry (cf. [S 1] for a definition). In fact, if $s$ is the degree of the nondegeneracy of $V$ one has $s+\ell=v+1$.

Let $M$ be a compact real submanifold of real dimension $2 p+1$. For $x \in M$, set $T_{x}^{1,0}(M)=T_{x}^{1,0}(V) \cap\left(T_{x}(M) \otimes \mathbb{C}\right)$. Then $T^{1,0}(M)$ is a subbundle of $T(M) \otimes \mathbb{C}$. We say that $\left(M, T^{1,0}(M)\right)$ is a $C R$ submanifold of $V$ with CR structure $T^{1,0}(M)$ if the following are satisfied:

(i) $\operatorname{dim}_{\mathbb{C}} T^{1,0}(M)=p$.

(ii) $T^{1,0}(M) \cap T^{0,1}(M)=\{0\}$, where $T^{0,1}(M)=\overline{T^{1,0}(M)}$.

(iii) For any two sections $X_{1}, X_{2}$ of $T^{1,0}(M)$ defined on an open set $U \subset M$, the Lie bracket $\left[X_{1}, X_{2}\right]$ is a section of $T^{1,0}(M)$ defined on $U$.

Let $X_{1}, \ldots, X_{p}$ be a local framing of $T^{1,0}(M)$ on an open set $U$. Then $\bar{X}_{1}, \ldots, \bar{X}_{p}$ is a local framing of $T^{0,1}(M)$ on $U$. Choose a section $T$ of $T(M) \otimes$ 
$\mathbb{C}$ so that $X_{1}, \ldots, X_{p}, \bar{X}_{1}, \ldots, \bar{X}_{p}, T$ gives a framing of $T(M) \otimes \mathbb{C}$ on $U$. Define a hermitian matrix $\left(c_{i j}\right)$ by:

$$
\left[X_{i}, \bar{X}_{j}\right]=c_{i j} T, \bmod X_{1}, \ldots, X_{p}, \bar{X}_{1}, \ldots, \bar{X}_{p}
$$

The hermitian form $\tau$ determined by $\left(c_{i j}\right)$ is called the Levi form of the $C R$ structure. The Levi matrix $\left(c_{i j}\right)$ clearly depends on the choice of framing however the rank and nullity of the associated form $\tau$ are well-defined. Throughout this paper we will call the complex dimension of the null space of the Levi form of $M$ the nullity of $M$.

By studying the relative geometry of a pair of submanifolds of $\mathbb{P}^{v}$ consisting of a compact $\mathrm{CR}$ submanifold and a compact complex submanifold we prove the following Lefschetz theorems for compact CR submanifolds with degenerate Levi form.

Theorem 2.1. Let $M$ be a compact $C R$ submanifold of $\mathbb{P}^{v}$ of dimension $2 p+1$. Suppose that the nullity of $M$ is everywhere greater than or equal to $r$, where $0<r \leq p$. If $j \leq r-1$ then

$$
\pi_{j}\left(M, M \cap \mathbb{P}^{v-1}\right)=0 .
$$

Hence, if $j<r-1$ then the map induced by inclusion

$$
\pi_{j}\left(M \cap \mathbb{P}^{v-1}\right) \rightarrow \pi_{j}(M),
$$

is an isomorphism and if $j=r-1$ this map is onto.

Note that, for generic $\mathbb{P}^{v-1} \subset \mathbb{P}^{v}, M \cap \mathbb{P}^{v-1}$ is a compact $\mathrm{CR}$ submanifold of $\mathbb{P}^{v-1}$ of dimension $2(p-1)+1$ with nullity everywhere greater than or equal to $r-1$, where $0<r-1 \leq p-1$. Therefore the result can be iterated.

We say that $M$ is Levi flat if the Levi form of the CR structure vanishes. In particular, a Levi flat submanifold admits a codimension one foliation with complex analytic leaves.

Corollary 2.2. Let $M$ be a compact Levi flat submanifold of $\mathbb{P}^{v}$ of dimension $m=2 p+1$. If $j \leq p-1$ then

$$
\pi_{j}\left(M, M \cap \mathbb{P}^{v-1}\right)=0 .
$$

Hence, if $j<p-1$ then the map induced by inclusion

$$
\pi_{j}\left(M \cap \mathbb{P}^{v-1}\right) \rightarrow \pi_{j}(M),
$$

is an isomorphism and if $j=p-1$ this map is onto. 
For generic $\mathbb{P}^{v-1}, M \cap \mathbb{P}^{v-1}$ is a compact Levi flat submanifold of $\mathbb{P}^{v-1}$ of dimension $2(p-1)+1$ and so, as above, the result can be iterated. Combining Corollary 2.2 with the Hurewicz isomorphism theorem we get the homology version of the Lefschetz theorem on hyperplane sections for Levi flat submanifolds of $\mathbb{P}^{v}$.

By studying the relative geometry of a pair of compact CR submanifolds of a compact hermitian symmetric space $V$ with $\operatorname{dim}_{\mathbb{C}} V=v$ we derive restrictions on the topology of a compact $\mathrm{CR}$ submanifold with degenerate Levi form.

Theorem 2.3. Let $V$ be an irreducible compact Kähler manifold of complex dimension $v$ with nonnegative holomorphic bisectional curvature of complex positivity $\ell$. Let $M$ be a $C R$ submanifold of real dimension $2 p+1$ and suppose that the nullity of $M$ is everywhere greater than or equal to $r$, where $0<r \leq p$. Then,

$$
\pi_{j}(V, M)=0,
$$

provided $j \leq \ell+1-2(v-r)$.

In particular if $M$ is Levi flat of real dimension $m$ and $m \geq 2(v+1)-\ell$ then it follows that $M$ is simply connected. The next result then follows from a theorem of Haefliger $[\mathrm{H}]$.

Theorem 2.4. Let $V$ be an irreducible compact Kähler manifold of complex dimension $v$ with nonnegative holomorphic bisectional curvature of complex positivity $\ell$. There are no real analytic Levi flat submanifolds of dimension $m$ in $V$ when $m \geq 2(v+1)-\ell$.

\section{The relative geometry of a complex submanifold and a CR submanifold.}

Let $V$ be an irreducible compact Kähler manifold of complex dimension $v$, with nonnegative bisectional curvature and with complex structure $J$. Let $\ell$ be the complex positivity of $V$. Let $M$ be a real smooth compact submanifold of dimension $2 p+1$ and let $N$ be a compact complex submanifold of complex dimension $n$.

We denote, by $\Omega(V ; M, N)=\Omega$, the space of piecewise smooth paths $\gamma:[0,1] \rightarrow V$ constrained by the requirements that $\gamma(0) \in M, \gamma(1) \in N$. Consider the energy of a path

$$
E(\gamma)=\int_{0}^{1}|\dot{\gamma}|^{2} d t
$$


as a function on $\Omega$. It is shown in $[\mathrm{S}-\mathrm{W}]$ that $\gamma$ is a critical point of $E$ if:

(i) $\gamma$ is a smooth geodesic

(ii) $\gamma$ is normal to $M$ at $\gamma(0)$ and normal to $N$ at $\gamma(1)$, respectively.

Let $W_{1}, W_{2} \in T_{\gamma} \Omega$. If $\gamma$ is a critical point of $E$ then the second variation of $E$ along $\gamma$ is:

$$
\begin{aligned}
\frac{1}{2} E_{* *}\left(W_{1}, W_{2}\right)= & \left.\left\langle\nabla_{W_{1}} W_{2}, \dot{\gamma}\right\rangle\right|_{0} ^{1}+\int_{0}^{1}\left\langle\nabla_{\dot{\gamma}} W_{1}, \nabla_{\dot{\gamma}} W_{2}\right\rangle d t \\
& -\int_{0}^{1}\left\langle R\left(\dot{\gamma}, W_{1}\right) \dot{\gamma}, W_{2}\right\rangle d t .
\end{aligned}
$$

where $R$ denotes the curvature tensor of $V$.

Theorem 3.1. Let $M$ be a $C R$ submanifold in $V$ of dimension $2 p+1$. Suppose that the nullity of $M$ is everywhere greater than or equal to $r$, where $0<r \leq p$. Let $N$ be a compact complex submanifold of complex dimension $n$. Then the index of a critical point $\gamma$ of $E$ is at least $\ell+1-(v-r)-(v-n)$, where $\ell$ is the complex positivity of $V$.

Proof. Since the null space of the Levi form of $M$ everywhere has dimension greater than or equal to $r$, for any point $x \in M$, locally there are $\mathbb{C}$-linearly independent vector fields $X_{1}, X_{2}, \ldots, X_{r}$ that are sections of $T^{1,0}(M)$ and that lie in the null space of $\tau$. We can choose the $X_{i}$ so that if $X_{i}=$ $W_{i}-\sqrt{-1} J W_{i}$ then $W_{1}, \ldots, W_{r}, J W_{1}, \ldots, J W_{r}$ are orthonormal. Now we follow the argument of $[\mathrm{S}-\mathrm{W}]$ and estimate the index of a critical point $\gamma$ of E.

By the second variation formula we have that

$$
\begin{aligned}
\frac{1}{2} E_{* *}(W, W)= & <\nabla_{W} W, \dot{\gamma}>\left.\right|_{0} ^{1}+\int_{0}^{1}<\nabla_{\dot{\gamma}} W, \nabla_{\dot{\gamma}} W>d t \\
& -\int_{0}^{1}<R(\dot{\gamma}, W) \dot{\gamma}, W>d t .
\end{aligned}
$$

Parallel translate $W_{i}, i=1, \ldots, r$, along $\gamma$ to construct a vector field $W_{i}(t)$ along $\gamma$. Of course, $W_{i}(1)$ need not be tangent to $N$ at $\gamma(1)$ so $W_{i}(t)$ is not necessarily an element of $T_{\gamma} \Omega$. However whenever $W_{i}(1) \in T N$ we have:

$$
\frac{1}{2} E_{* *}\left(W_{i}, W_{i}\right)=<\nabla_{W_{i}} W_{i}, \dot{\gamma}>\left.\right|_{0} ^{1}-\int_{0}^{1}<R\left(\dot{\gamma}, W_{i}\right) \dot{\gamma}, W_{i}>d t
$$


Similarly, parallel translate $J W_{i}$ along $\gamma$ to get:

$$
\frac{1}{2} E_{* *}\left(J W_{i}, J W_{i}\right)=<\nabla_{J W_{i}} J W_{i}, \dot{\gamma}>\left.\right|_{0} ^{1}-\int_{0}^{1}<R\left(\dot{\gamma}, J W_{i}\right) \dot{\gamma}, J W_{i}>d t .
$$

Observe that,

$$
\begin{aligned}
<\nabla_{J W_{i}} J W_{i}, \dot{\gamma}>= & <J\left(\nabla_{J W_{i}} W_{i}\right), \dot{\gamma}> \\
= & -<\nabla_{J W_{i}} W_{i}, J \dot{\gamma}> \\
= & -<\nabla_{W_{i}} J W_{i}, J \dot{\gamma}>+<\left[J W_{i}, W_{i}\right], J \dot{\gamma}> \\
= & -<\nabla_{W_{i}} W_{i}, \dot{\gamma}>+<\left[J W_{i}, W_{i}\right], J \dot{\gamma}>,
\end{aligned}
$$

At $\gamma(0)$ the vector $\dot{\gamma}$ is normal to $M$. Thus at $\gamma(0)$ the vector $J \dot{\gamma}$ is either normal to $M$ or, if tangent to $M$, then it is normal to $T^{1,0}(M) \oplus T^{0,1}(M)$. By assumption $X_{i}(0)$ lies in the null space of the Levi form, so at $\gamma(0)$, $<\left[J W_{i}, W_{i}\right], J \dot{\gamma}>=0$. To show that $\left\langle\left[J W_{i}, W_{i}\right], J \dot{\gamma}>=0\right.$ at $\gamma(1)$ we use a simple argument of Frankel $[\mathrm{F}]$. Let $R$ be an analytic curve that passes through $\gamma(1)$ with tangent vectors $W_{i}, J W_{i}$ at $\gamma(1)$ and extend $W_{i}, J W_{i}$ to be tangent vector fields to $R$ in a neighborhood $U$ of $\gamma(1)$. Then on $U,\left[J W_{i}, W_{i}\right]$ and $J\left[J W_{i}, W_{i}\right]$ are tangent to $R$ and so, at $\gamma(1)$, they are perpendicular to $\dot{\gamma}(1)$.

Therefore adding (3.1) and (3.2) we have

$$
\begin{aligned}
\frac{1}{2} E_{* *}\left(W_{i}, W_{i}\right) & +\frac{1}{2} E_{* *}\left(J W_{i}, J W_{i}\right)= \\
& -\int_{0}^{1}\left(<R\left(\dot{\gamma}, W_{i}\right) \dot{\gamma}, W_{i}>+<R\left(\dot{\gamma}, J W_{i}\right) \dot{\gamma}, J W_{i}>\right) d t .
\end{aligned}
$$

Using the symmetries of the curvature tensor we have:

$$
\left\langle R\left(\dot{\gamma}, W_{i}\right) \dot{\gamma}, W_{i}\right\rangle+\left\langle R\left(\dot{\gamma}, J W_{i}\right) \dot{\gamma}, J W_{i}\right\rangle=\left\langle R(\dot{\gamma}, J \dot{\gamma}) W_{i}, J W_{i}\right\rangle .
$$

This expression is the holomorphic bisectional curvature of the complex lines $\dot{\gamma} \wedge J \dot{\gamma}$ and $W_{i} \wedge J W_{i}$.

Note that the vectors $W_{i}(1), J W_{i}(1)$ are perpendicular to both $\dot{\gamma}(1)$ and $J \dot{\gamma}(1)$. Thus the vector space

$$
S=\operatorname{span}\left\{W_{1}(1), \ldots, W_{r}(1), J W_{1}(1), \ldots, J W_{r}(1)\right\}
$$

is a complex $r$-dimensional space lying in a complex $(v-1)$-dimensional subspace of $T_{\gamma(1)} V$. It follows that the subspace $S \cap T_{\gamma(1)} N$ has complex dimension at least equal to

$$
r+n-(v-1)
$$


Moreover, the vector fields $\{W, J W\}$ with $W(1), J W(1) \in S \cap T_{\gamma(1)} N$ are parallel and lie in $T_{\gamma} \Omega$. Therefore under the assumption that the positivity of $V$ is $\ell$ it follows that every nontrivial critical point $\gamma$ of $E$ on $\Omega$ has index $\lambda \geq \lambda_{0}$ with,

$$
\lambda_{0}=r+n-(v-1)-(v-\ell) .
$$

Using the relation between Morse theory on the path space $\Omega$ and the topology of $(V ; M, N)$ (see $[\mathrm{S}-\mathrm{W}]$ or $[\mathrm{K}-\mathrm{W}]$ for details) we have:

Theorem 3.2. Let $V$ be a compact Kähler manifold of non-negative holomorphic bisectional curvature, of complex dimension $v$ and with complex positivity $\ell$. Let $M$ be a compact $C R$ submanifold of $V$ of dimension $m=2 k+1$ and suppose that the nullity of $M$ is everywhere greater than or equal to $r$, where $0<r \leq k$. Let $N$ be a compact complex submanifold of complex dimension $n$. Then the homomorphism induced by the inclusion

$$
\imath_{*}: \pi_{j}(M, M \cap N) \rightarrow \pi_{j}(V, N)
$$

is an isomorphism for $j \leq n+r-v-(v-\ell)$ and is a surjection for $j=$ $n+r-v-(v-\ell)+1$.

The following result is proved in $[\mathrm{S}-\mathrm{W}]$ and $[\mathrm{K}-\mathrm{W}]$ using techniques analogous to those used above.

Theorem 3.3. Let $V$ be a compact Kähler manifold of non-negative holomorphic bisectional curvature, of complex dimension $v$ and with complex positivity $\ell$. Let $N$ be a compact complex submanifold of complex dimension $n$. If

$$
j \leq 2 n-v-(v-\ell)+1
$$

then

$$
\pi_{j}(V, N)=0
$$

Corollary 3.4. Under the same hypothesis as in Theorem 3.2, if

$$
j \leq \min (2 n-v-(v-\ell)+1, n+r-v-(v-\ell))
$$

then

$$
\pi_{j}(M, M \cap N)=0 .
$$


Proof. Follows from Theorem 3.3 and Theorem 3.2.

Applying Corollay 3.4 to the special case when $V=\mathbb{P}^{v}$ with the FubiniStudy metric and $N=\mathbb{P}^{v-1}$ is a hyperplane we have the Lefschetz hyperplane theorem for compact CR submanifolds with degenerate Levi form (Theorem 2.1) and for Levi flat submanifolds (Corollary 2.2).

\section{The relative geometry of a pair of CR submanifolds.}

Let $V$ be an irreducible compact Kähler manifold of complex dimension $v$, with nonnegative bisectional curvature and with complex structure $J$. Let $\ell$ be the complex positivity of $V$. Let $M$ and $N$ be a real smooth compact submanifolds of dimensions $m=2 p+1$ and $n=2 q+1$, respectively.

We denote, by $\Omega(V ; M, N)=\Omega$, the space of piecewise smooth paths $\gamma:[0,1] \rightarrow V$ constrained by the requirements that $\gamma(0) \in M, \gamma(1) \in N$. Consider the energy of a path

$$
E(\gamma)=\int_{0}^{1}|\dot{\gamma}|^{2} d t
$$

as a function on $\Omega$. It is shown in $[\mathrm{S}-\mathrm{W}]$ that $\gamma$ is a critical point of $E$ if:

(i) $\gamma$ is a smooth geodesic

(ii) $\gamma$ is normal to $M$ at $\gamma(0)$ and normal to $N$ at $\gamma(1)$, respectively.

Let $W_{1}, W_{2} \in T_{\gamma} \Omega$. If $\gamma$ is a critical point of $E$ then the second variation of $E$ along $\gamma$ is:

$$
\begin{aligned}
\frac{1}{2} E_{* *}\left(W_{1}, W_{2}\right)= & \left.\left\langle\nabla_{W_{1}} W_{2}, \dot{\gamma}\right\rangle\right|_{0} ^{1}+\int_{0}^{1}\left\langle\nabla_{\dot{\gamma}} W_{1}, \nabla_{\dot{\gamma}} W_{2}\right\rangle d t \\
& -\int_{0}^{1}\left\langle R\left(\dot{\gamma}, W_{1}\right) \dot{\gamma}, W_{2}\right\rangle d t .
\end{aligned}
$$

where $R$ denotes the curvature tensor of $V$.

Theorem 4.1. Let $M$ and $N$ be $C R$ submanifolds of $V$ of dimensions $2 p+1$ and $2 q+1$, respectively. Suppose that the nullity of $M$ and $N$ are everywhere greater than or equal to $r$ and $s$, respectively, where $0<r \leq p$ and $0<s \leq q$. Then the index of a critical point $\gamma$ of $E$ is at least $\ell+1-(v-r)-(v-s)$, where $\ell$ is the complex positivity of $V$. 
Proof. Since the null space of the Levi form of $M$ everywhere has dimension greater than or equal to $r$, for any point $x \in M$, locally there are $\mathbb{C}$-linearly independent vector fields $X_{1}, X_{2}, \ldots, X_{r}$ that are sections of $T^{1,0}(M)$ and that lie in the null space of the Levi form of $M$. We can choose the $X_{i}$ so that if $X_{i}=W_{i}-\sqrt{-1} J W_{i}$ then $W_{1}, \ldots, W_{r}, J W_{1}, \ldots, J W_{r}$ are orthonormal. Now we follow the argument of $[\mathrm{S}-\mathrm{W}]$ and estimate the index of a critical point $\gamma$ of $E$.

By the second variation formula we have that

$$
\begin{aligned}
\frac{1}{2} E_{* *}(W, W)= & <\nabla_{W} W, \dot{\gamma}>\left.\right|_{0} ^{1}+\int_{0}^{1}<\nabla_{\dot{\gamma}} W, \nabla_{\dot{\gamma}} W>d t \\
& -\int_{0}^{1}<R(\dot{\gamma}, W) \dot{\gamma}, W>d t .
\end{aligned}
$$

Parallel translate $W_{i}, i=1, \ldots, r$, along $\gamma$ to construct a vector field $W_{i}(t)$ along $\gamma$. Of course, $W_{i}(1)$ need not be tangent to $N$ at $\gamma(1)$ so $W_{i}(t)$ is not necessarily an element of $T_{\gamma} \Omega$. However whenever $W_{i}(1) \in T N$ we have:

$$
\frac{1}{2} E_{* *}\left(W_{i}, W_{i}\right)=<\nabla_{W_{i}} W_{i}, \dot{\gamma}>\left.\right|_{0} ^{1}-\int_{0}^{1}<R\left(\dot{\gamma}, W_{i}\right) \dot{\gamma}, W_{i}>d t .
$$

Similarly, parallel translate $J W_{i}$ along $\gamma$ to get:

$$
\frac{1}{2} E_{* *}\left(J W_{i}, J W_{i}\right)=<\nabla_{J W_{i}} J W_{i}, \dot{\gamma}>\left.\right|_{0} ^{1}-\int_{0}^{1}<R\left(\dot{\gamma}, J W_{i}\right) \dot{\gamma}, J W_{i}>d t .
$$

Now assume that $X_{i}(1)=W_{i}(1)-\sqrt{-1} J W_{i}(1)$ lies in the null space of the Levi form of $N$ at $\gamma(1)$. Observe that,

$$
\begin{aligned}
<\nabla_{J W_{i}} J W_{i}, \dot{\gamma}>= & <J\left(\nabla_{J W_{i}} W_{i}\right), \dot{\gamma}> \\
= & -<\nabla_{J W_{i}} W_{i}, J \dot{\gamma}> \\
= & -<\nabla_{W_{i}} J W_{i}, J \dot{\gamma}>+<\left[J W_{i}, W_{i}\right], J \dot{\gamma}> \\
= & -<\nabla_{W_{i}} W_{i}, \dot{\gamma}>+<\left[J W_{i}, W_{i}\right], J \dot{\gamma}>,
\end{aligned}
$$

At $\gamma(0)$ the vector $\dot{\gamma}$ is normal to $M$. Thus at $\gamma(0)$ the vector $J \dot{\gamma}$ is either normal to $M$ or, if tangent to $M$, then it is normal to $T^{1,0}(M) \oplus T^{0,1}(M)$. By assumption $X_{i}(0)$ lies in the null space of the Levi form, so at $\gamma(0)$, $<\left[J W_{i}, W_{i}\right], J \dot{\gamma}>=0$. To show that $<\left[J W_{i}, W_{i}\right], J \dot{\gamma}>=0$ at $\gamma(1)$ we use the assumption that $X_{i}(1)$ lies in the null space of the Levi form of $N$ at 
$\gamma(1)$ and apply the previous reasoning. Therefore adding (2.1) and (2.2) we have

$$
\begin{aligned}
\frac{1}{2} E_{* *}\left(W_{i}, W_{i}\right) & +\frac{1}{2} E_{* *}\left(J W_{i}, J W_{i}\right)= \\
& -\int_{0}^{1}\left(<R\left(\dot{\gamma}, W_{i}\right) \dot{\gamma}, W_{i}>+<R\left(\dot{\gamma}, J W_{i}\right) \dot{\gamma}, J W_{i}>\right) d t
\end{aligned}
$$

Using the symmetries of the curvature tensor we have:

$$
\left\langle R\left(\dot{\gamma}, W_{i}\right) \dot{\gamma}, W_{i}\right\rangle+\left\langle R\left(\dot{\gamma}, J W_{i}\right) \dot{\gamma}, J W_{i}\right\rangle=\left\langle R(\dot{\gamma}, J \dot{\gamma}) W_{i}, J W_{i}\right\rangle .
$$

This expression is the holomorphic bisectional curvature of the complex lines $\dot{\gamma} \wedge J \dot{\gamma}$ and $W_{i} \wedge J W_{i}$.

Note that the vectors $W_{i}(1), J W_{i}(1)$ are perpendicular to both $\dot{\gamma}(1)$ and $J \dot{\gamma}(1)$. Thus the vector space

$$
S=\operatorname{span}\left\{W_{1}(1), \ldots, W_{r}(1), J W_{1}(1), \ldots, J W_{r}(1)\right\}
$$

is a complex $r$-dimensional space lying in a complex $(v-1)$-dimensional subspace of $T_{\gamma(1)} V$. Denote the null space of the Levi form of $N$ at $x \in N$ by $\mathcal{Z}_{x}(T N)$. Then the subspace $S \cap \mathcal{Z}_{\gamma(1)}(T N)$ has complex dimension at least equal to

$$
r+s-(v-1) .
$$

Moreover, the vector fields $\{W, J W\}$ with $W(1), J W(1) \in S \cap \mathcal{Z}_{\gamma(1)}(T N)$ are parallel and lie in $T_{\gamma} \Omega$. Therefore under the assumption that the positivity of $V$ is $\ell$ it follows that every nontrivial critical point $\gamma$ of $E$ on $\Omega$ has index $\lambda \geq \lambda_{0}$ with,

$$
\lambda_{0}=r+s-(v-1)-(v-\ell)
$$

Using the relation between Morse theory on the path space $\Omega$ and the topology of $(V ; M, N)$ (see $[\mathrm{S}-\mathrm{W}]$ or $[\mathrm{K}-\mathrm{W}]$ for details) we have:

Theorem 4.2. Let $V$ be a compact Kähler manifold of non-negative holomorphic bisectional curvature, of complex dimension $v$ and with complex positivity $\ell$. Let $M, N$ be a compact $C R$ submanifolds of $V$ of dimensions $2 p+1$ and $2 q+1$, respectively and suppose that the nullity of $M$ and $N$ are everywhere greater than or equal to $r$ and $s$, respectively, where $0<r \leq p$ and $0<s \leq q$. Then the homomorphism induced by the inclusion

$$
\imath_{*}: \pi_{j}(M, M \cap N) \rightarrow \pi_{j}(V, N)
$$


is an isomorphism for $j \leq r+s-v-(v-\ell)$ and is a surjection for $j=$ $r+s-v-(v-\ell)+1$.

Applying this result to the case $M=N$ we have:

Corollary 4.3. Let $M$ be a $C R$ submanifold of $V$ as in Theorem 4.2 . Then

$$
\pi_{j}(V, M)=0
$$

provided $j \leq \ell+1-2(v-r)$, where $\ell$ is the complex positivity of $V$.

Using the exact homotopy sequence of the pair $(V, M)$ and that $\pi_{1}(V)=$ 0 we conclude:

Corollary 4.4. Let $V$ and $M$ be as above. If $2 r \geq 2 v+1-\ell$ then $\pi_{1}(M)=$ $\{0\}$.

Corollary 4.5. Let $V$ be as above and suppose that $M$ is Levi flat. If $\operatorname{dim} M \geq 2(v+1)-\ell$ then $\pi_{1}(M)=\{0\}$.

Recall the well known result of A. Haefliger $[\mathrm{H}]$.

Theorem 4.6. There is no real analytic, codimension one foliation of a compact simply-connected manifold.

Corollary 4.5 and Theorem 4.6 imply Theorem 2.4 .

Combining the topological restrictions imposed on Levi flat submanifolds by Theorem 4.2 and its corollaries with codimension one foliation theory leads to many partial nonexistence results such as:

Theorem 4.7. Let $V$ be an irreducible compact Kähler manifold of complex dimension $v$ with complex positivity $\ell$. If $m \geq 2(v+1)-\ell$ then there are no smooth Levi flat submanifolds of dimension $m$ in $V$ with a compact leaf.

We leave the formulation of similar results to the reader.

\section{References.}

[B] Borel, A., On the curvature tensor of the Hermitian symmetric manifolds, Ann. of Math. 71 (1960), 508-521.

[C-V] Calabi, E., and Vesentini, E., On compact locally symmetric Kähler manifolds, Ann. of Math. 71 (1960), 472-507. 
[C-S] Chen, S., and Shaw, M., Partial differential equations in several complex variables, AMS and International Press, 2001.

[H] Haefliger, A., Variétés feuilletées, Ann. Scuola Norm. Sup. Pisa, 16 (1962), 367-397.

[F] Frankel, T., Manifolds with Positive Curvature, Pacific J. 11 (1961), $165-174$.

[K-W] Kim, M., and Wolfson, J., Theorems of Barth-Lefschetz type on Kähler manifolds of non-negative bisectional curvature, Forum Math. 15 (2003), 261-273.

[LN] Lins Neto, A., A note on projective Levi-flats and minimal sets of algebraic functions, Ann. Inst. Fourier 49 (1999), 1369-1385.

[M] Mok, N., The uniformization theorem for compact Kähler manifolds of nonnegative holomorphic bisectional curvature, J. Diff. Geom. 27 (1988), 179-214.

[O] Ohsawa, T ., Non-existence of real analytic Levi-flat hypersurfaces in $\mathbb{P}^{2}$, Nagoya Math J., 158 (2000), 95-98.

[S-W] Schoen, R., and Wolfson, J., Theorems of Barth-Lefschetz type and Morse theory on the space of paths, Math Z. 229 (1998), 77-87.

[S 1] Siu, Y-T., Nonexistence of smooth Levi-flat hypersurfaces in complex projective spaces of dimension $\geq 3$, Ann. of Math. 151 1217-1243, (2000).

[S 2] Siu, Y-T., $\bar{\partial}$-Regularity for weakly pseudoconvex domains in compact Hermitian symmetric spaces with respect to invariant metrics, Ann. of Math. 156 595-621, (2002).

Received September 4, 2002. 